\title{
Insulin resistance in spontaneous obese Rhesus monkeys (Macaca mulatta) - A valuable animal model
}

\author{
Charon de Villiers ${ }^{* 1}$, Joritha van Heerden ${ }^{1}$ and Johan Louw ${ }^{2}$ \\ 1*PUDAC - Primate Unit and Delft Animal Centre, South African Medical Research Council, Cape Town, South Africa \\ ${ }^{2}$ BRIP - Biomedical Research and Innovation Platform, South African Medical Research Council, Cape Town, South Africa
}

*Correspondence to: Dr. Charon de Villiers, PUDAC-Delft Animal Facility, South African Medical Research Council, Private Bag 19001, Tygerberg, Cape Town, South Africa, 7505; Tel: +27 219380671 Mobile: +27 723852716; E-mail: charon.deVilliers@mrc.ac.za

Received: November 27, 2017; Accepted: December 05, 2017; Published: December 12, 2017;

\begin{abstract}
Background: The epidemiology of Type 2 diabetes mellitus (T2D) in humans is reported with older individuals presenting increased body weight. As in humans, non-human primates have obesity-associated insulin resistance that is initially met with compensatory insulin secretion.

Methods: A group of adult Rhesus females (Macaca mulatta), among a colony of 70 captive-bred Rhesus monkeys, were identified with spontaneous obesity. Measurements of body weights, crown-rump lengths, and abdominal circumferences were recorded from ( $\mathrm{n}=9$ ) obese animals and also from $(n=9)$ lean females, to serve as a control. To test insulin resistance, both groups were subjected to an intravenous glucose tolerance test (IVGTT).
\end{abstract}

Results: Both groups indicated a similar glucose uptake but with significantly higher levels of insulin in the obese group.

Conclusion: The higher insulin and the fact that glucose levels were normal in the two groups after IVGGT, is a reflection of compensatory hyperinsulinemia among the obese group. We propose this group of Rhesus monkeys to be the ideal model to study obesity and other chronic diseases associated with T2D.

Keywords: female Rhesus monkeys, non-human primates, obesity, type 2 diabetes (T2D), glucose, insulin

\section{Introduction}

Type 2 diabetes (T2D), and its complications such as cardiovascular and kidney disease, largely driven by obesity, now represents the major non-communicable disease burden worldwide. T2D is also associated with specific dyslipidaemia (elevated triglycerides, reduced HDL cholesterol, and increased small, dense low-density lipoprotein (LDL) particles) that further increases the risk of developing cardiovascular disease. The likelihood and severity of T2D are closely linked with body mass index (BMI). It is reported that there are a seven times greater risk of diabetes in obese people compared to those of healthy weight, with a threefold increase in risk for overweight people [1]. Theories of why obesity may lead to T2D include: 1) abdominal obesity may cause fat cells to release pro-inflammatory chemicals. These chemicals can make the body less sensitive to the insulin it produces by disrupting the function of insulin responsive cells and their ability to respond to insulin $[2,3]$ and 2) obesity may trigger changes to the body's metabolism that cause adipose (fat) tissue to release increased amounts of fatty acids, glycerol, hormones, proinflammatory cytokines and other factors that are involved in the development of insulin resistance. When insulin resistance (IR) is accompanied by dysfunction of pancreatic islet beta cells it results in failure to control blood glucose levels [4].

To study the pathogenesis, evaluating appropriate interventions and identifying targets for therapy for this disease, suitable animal models are required. The ideal model for such studies would recreate the inherent history of the disease as it occurs in human populations. Therefore, such a model would exhibit natural variabilities in insulin resistance; some individuals become exacerbated at midlife under the condition of excessive caloric intake and lack of physical activity. Females would also be the ideal model with specific reference to cardiovascular disease, due to the hormonal profile [5]. Of all the animal models, diabetes has been reported in a number of Old World non-human primates such as the Cynomologus, Rhesus, African green monkey and the Baboon [6]. As with humans, T2D in nonhuman primates, such as the Rhesus monkey, is associated with obesity and increasing age [7]. In these animals, T2D was categorized into sequential phases based on age, body weight, glucose clearance, and fasting and secretory insulin levels [8]. Obese animals also exhibit increased fasting triglycerides and impaired insulin responses to glucose. Further investigations indicated that excess body fat was located primarily in the abdominal region, and glucoregulatory abnormalities were present in both sexes of obese Rhesus macaques. These findings in macaques have subsequently been noted to be similar to the central body fat distribution patterns in obese humans and their susceptibility to metabolic syndrome, including insulin resistance.

In laboratory housed monkeys, the peak body weight is usually not reached until about 15 years ('middle-age') with the weight gained after age seven years composed primarily of adipose tissue with a distribution mainly in the abdominal area. Reportedly, obesity in nonhuman primates is considered to be a disease of aging with individuals 
classified as obese when their body weight exceeds $15 \mathrm{~kg}$. However, even though non-human primates are able to reproduce by the age of four years there are no reports on spontaneous obesity in younger monkeys (under the age of six years). Therefore non-human primates do not provide a model for childhood-onset obesity in humans [9]. Obesity-related hyperinsulinemia and abnormal glucose tolerance have also been reported in feral Rhesus monkeys.

In non-human primates, diabetes includes a period of insulin resistance, with compensatory hyperinsulinemia despite normal glucose tolerance. This period of compensatory hyperinsulinemia is followed by a continued decrease of insulin secretory capacity. As the disease progresses, individuals develop compromised glucose tolerance with a slight increase in fasting glucose levels before advancing to hyperglycinaemia due to a virtual deterioration in pancreatic insulin secretion $[10,11]$.

With the increased incidence of human obesity and diabetes, animal models are especially relevant to study the interactions among obesity, IR, aging, and associated comorbidities. Diabetic non-human primates also have adverse changes in plasma lipid and lipoprotein concentrations, biomarkers of obesity, inflammation, and oxidative stress, and protein glycation that contribute to the numerous complications of the disease. Furthermore, sex hormones, pregnancy, and environmental factors (e.g., diet and stress) affect IR and can also contribute to diabetes progression in non-human primates [12]. These similarities between adult-onset, obesity-related diabetes in the Rhesus monkey and the human suggest further studies of these monkeys might provide useful and novel insights into the etiology of this disease. The purpose of this investigation was to confirm the relationship between obesity and glucoregulatory abnormalities in a group of captive-bred Rhesus females. The findings of this investigations will serve as supplementary data to information previously reported for the Rhesus monkey.

\section{Materials and Methods}

\section{Humane Care Guidelines}

Eighteen sexually mature captive-bred Rhesus females, with an average age of 15 years, were selected for this study. Nine individuals were identified as "obese" presenting body weights that ranged from $8,1 \mathrm{~kg}$ to $12,1 \mathrm{~kg}$. The remaining nine females were categorized as "lean" and revealed body weights ranging between $4,7 \mathrm{~kg}$ and $8,8 \mathrm{~kg}$. These females were selected from a free-ranging breeding colony, housed outdoors at the Delft Animal Centre, South African Medical Research Council (Cape Town, South Africa). The animals were maintained on a standard diet of special monkey cubes (Equifeeds, Cape Town, South Africa) and supplemented with seasonal fruit and vegetables. Additional enrichment devices were provided daily and water was available ad libitum. None of the females in the study had been used in other experimental studies. All animals were housed and maintained in accordance with the South African National Standard for the Care and Use of Animals for Scientific Purposes (The SANS 10386: 2008). The study was approved by the Ethics Committee for Research on Animals (ECRA) of the South African Medical Research Council.

\section{Measurements of body dimensions}

Somatometric measurements were recorded while animals were immobilized with Ketamine hydrochloride (Anaket V, Norpharm, Cape Town), at $10 \mathrm{mg} / \mathrm{kg}$. According to a body-condition scoring system [13], crown-rump (height) and crown-heel lengths were measured with the monkey in the supine position on a calibrated rule with a fixed headrest. The circumferences of the abdomen (at the level of the umbilicus) were taken with the monkey in lateral recumbency. The body weight of each animal was also recorded.

\section{Glucose tolerance tests and biochemical assays}

Monkeys were fasted overnight before the intravenous glucose tolerance tests (IVGTTs) and then sedated with Ketaminehydrochloride (Anaket V, Norpharm, Cape Town), at $10 \mathrm{mg} / \mathrm{kg}$. A graduated sterile catheter (Cavafix, Certo 257, B/Braun Melsungen AF) was inserted into the saphenous vein through a stainless steel trocar up to the 3,5 mark, according to the manufacturer's instructions. Thereafter, a sterile saline ( $0.9 \%$ sodium chloride, Norpharm, Cape Town) infusion line was immediately connected to the catheter to ensure patency after each blood collection. Baseline blood samples were collected for fasting glucose /insulin concentrations and glucagon determination in serum gel tubes sodium fluoride and EDTA tubes at -10 minutes prior to glucose administration. Intravenous glucose (DextroseFresenius, Norpharm, Cape Town), $50 \%$ at $1 \mathrm{~mL} / \mathrm{kg}$ was administered on the other limb over one minute followed by blood collections at time points 1, 3, 5, 10, 15, 20, 40 and 60 minutes. For glucose assay, blood was collected in tubes containing potassium oxylate/sodium fluoride and for insulin and lipids, blood was collected in serum gel tubes and EDTA tubes. To prevent elevated potassium levels during the biochemistry tests, particular care was taken during collections to avoid hemolysis of the blood samples. Plasma glucose was determined by the hexokinase method (Beckman Coulter AU Analyser). Fasting serum, total cholesterol, and triglycerides were determined by colorimetric analysis (PathCare, Laboratories, Cape Town, South Africa). Insulin was measured by a double immunoradiometric assay using human insulin as a standard and Rhesus serum for internal controls.

\section{Statistical analysis}

The difference between mean values of the lean group and the obese group was analyzed for significance using analysis of variance followed by the Newman-Keuls test. Significance between mean values from the two groups was determined with Student's $t$-test. Levels of $\mathrm{P}$ $<0.05$ were considered significant.

\section{Results}

\section{Somatometric Indices for body weight and measurements}

The high body weights observed from the obese individuals were primarily attributed to body fat rather than to stature or lean body mass (Figure 1) and waist circumference and abdominal fat fold (adipose fat) were also highly correlated with overall body fat (Figure 2). The obese group indicated an average body weight of 
$9,10 \mathrm{~kg}$ compared to the $6,15 \mathrm{~kg}$ body weight of the lean group. The crown-rump lengths were similar but results indicated a $48 \%$ weight distinction accommodated by a $43 \%$ difference in waist circumference between the 2 groups (Figure 3).

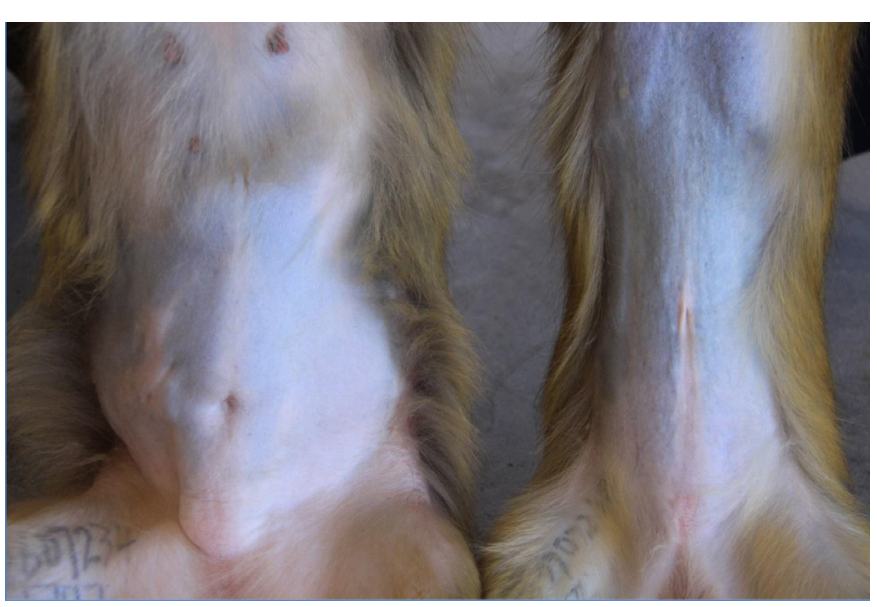

Figure 1. High body weight observed from an obese female (left) versus a lean female (right)

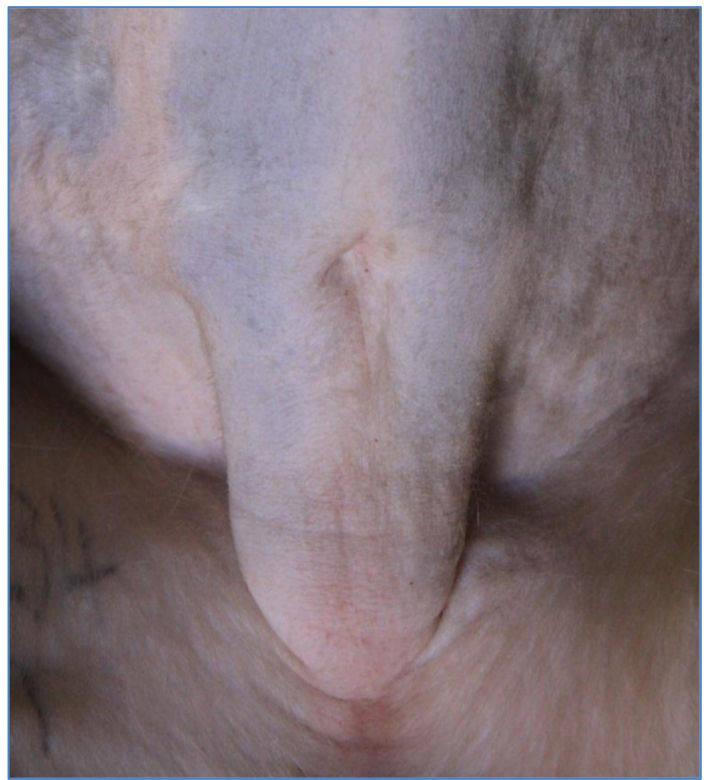

Figure 2. Waist circumference and abdominal fat fold (adipose fat)

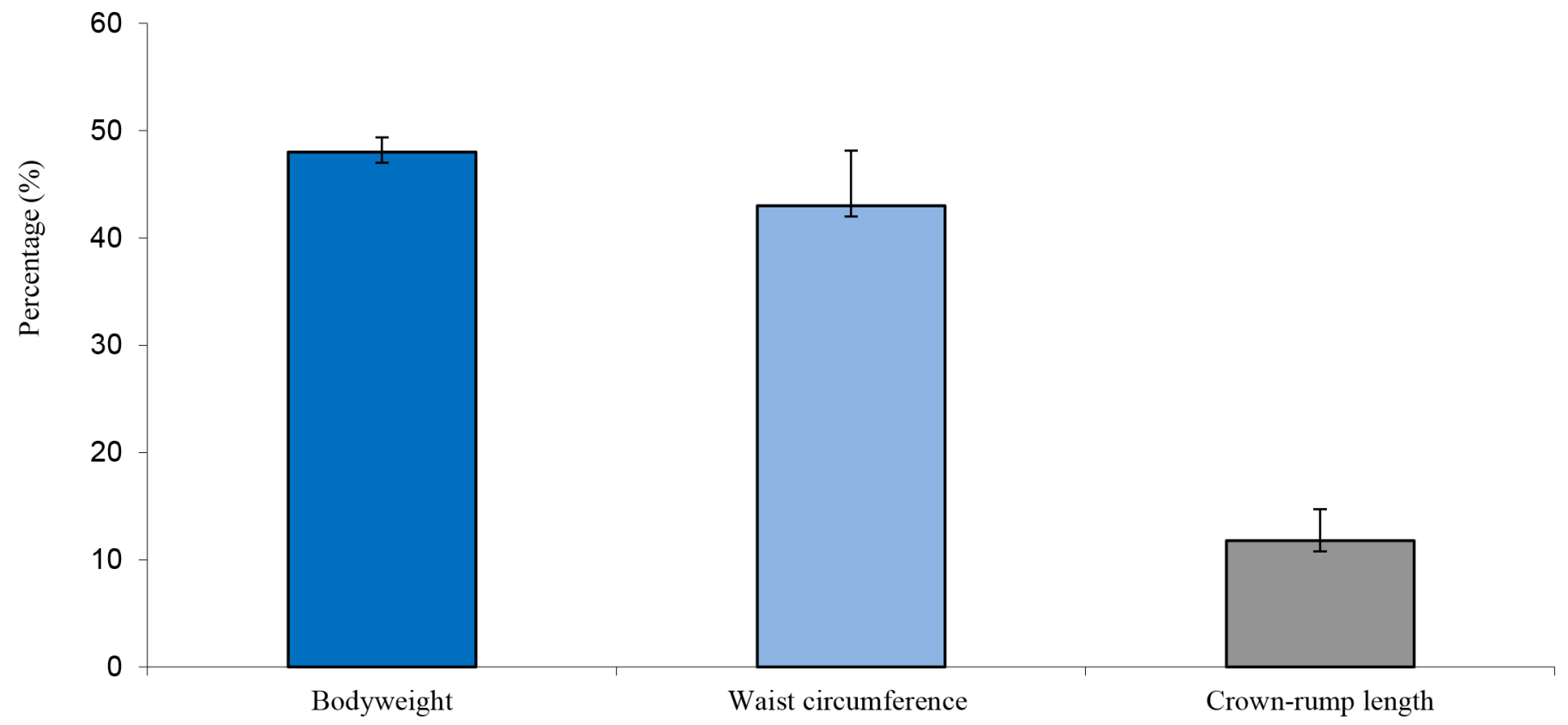

Figure 3. Average difference in weight, waist circumference and crown-rump length between obese and lean monkeys

\section{Glucose tolerance and insulin uptake}

The obese individuals indicated a similar glucose uptake compared to the lean controls, albeit with slightly more area under the curve (Figure 4 and Figure 5). However, insulin levels were significantly higher in the obese versus the lean individual $(\mathrm{P}<0.05)$.
The glucose and insulin values obtained during the IVGTTs indicated elevated insulin levels in the obese group but normal glucose levels for both groups. This observation is a reflection of compensatory hyperinsulinemia amongst the obese individuals (Figure 6 and Figure 7). 


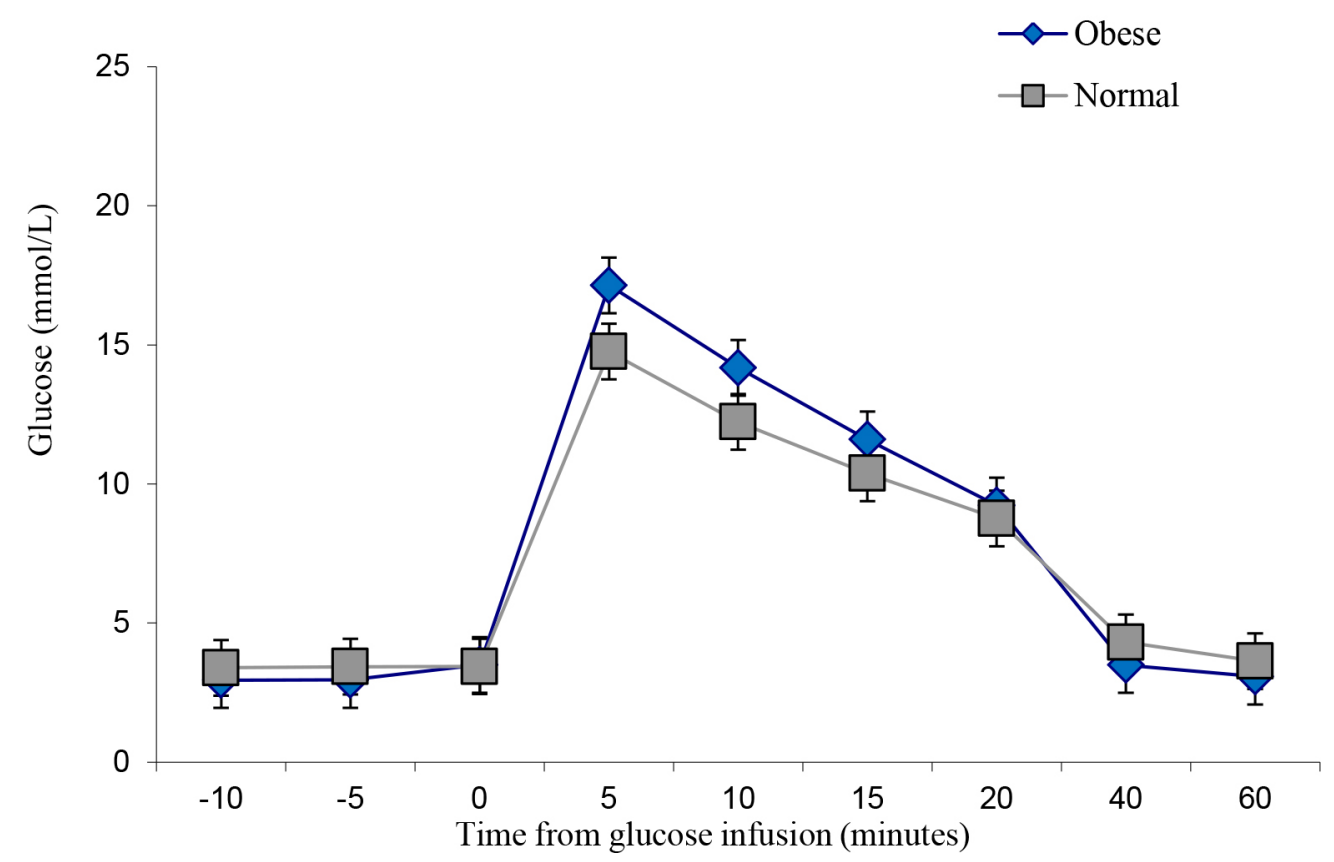

Figure 4. IVGTT Glucose levels

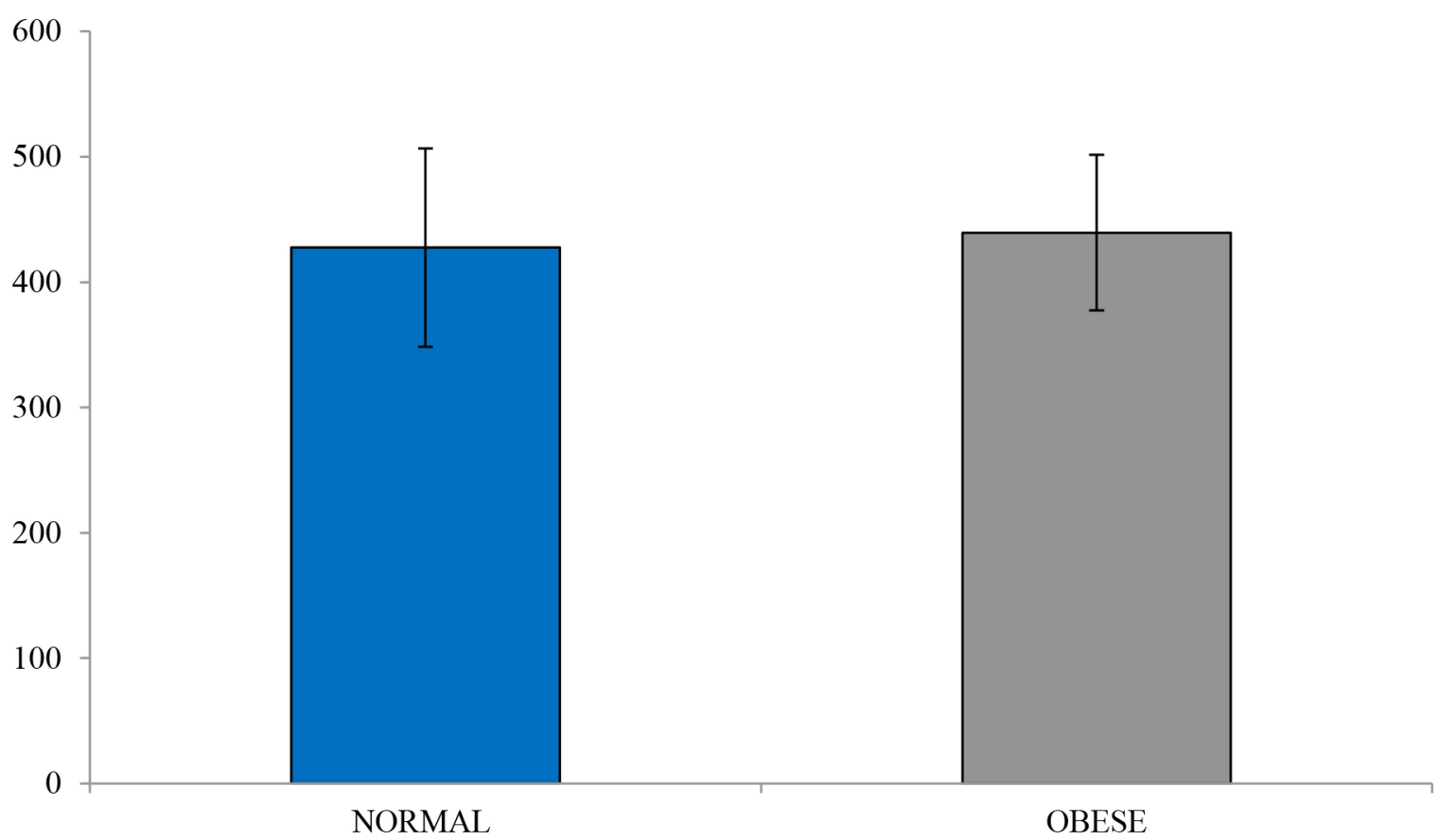

Figure 5. Glucose under the curve 


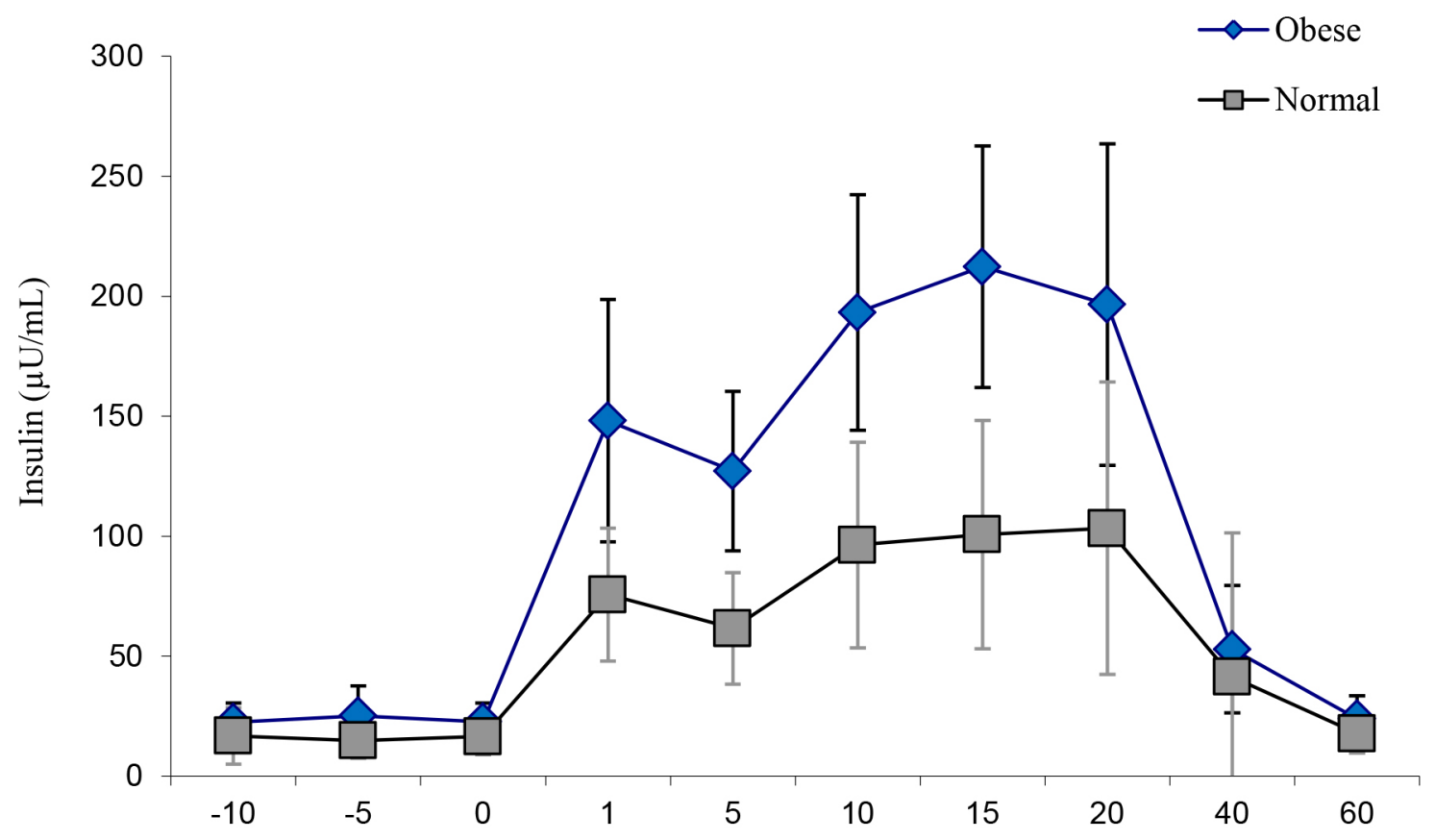

Time from glucose infusion (minutes)

Figure 6. IVGTT Insulin levels.
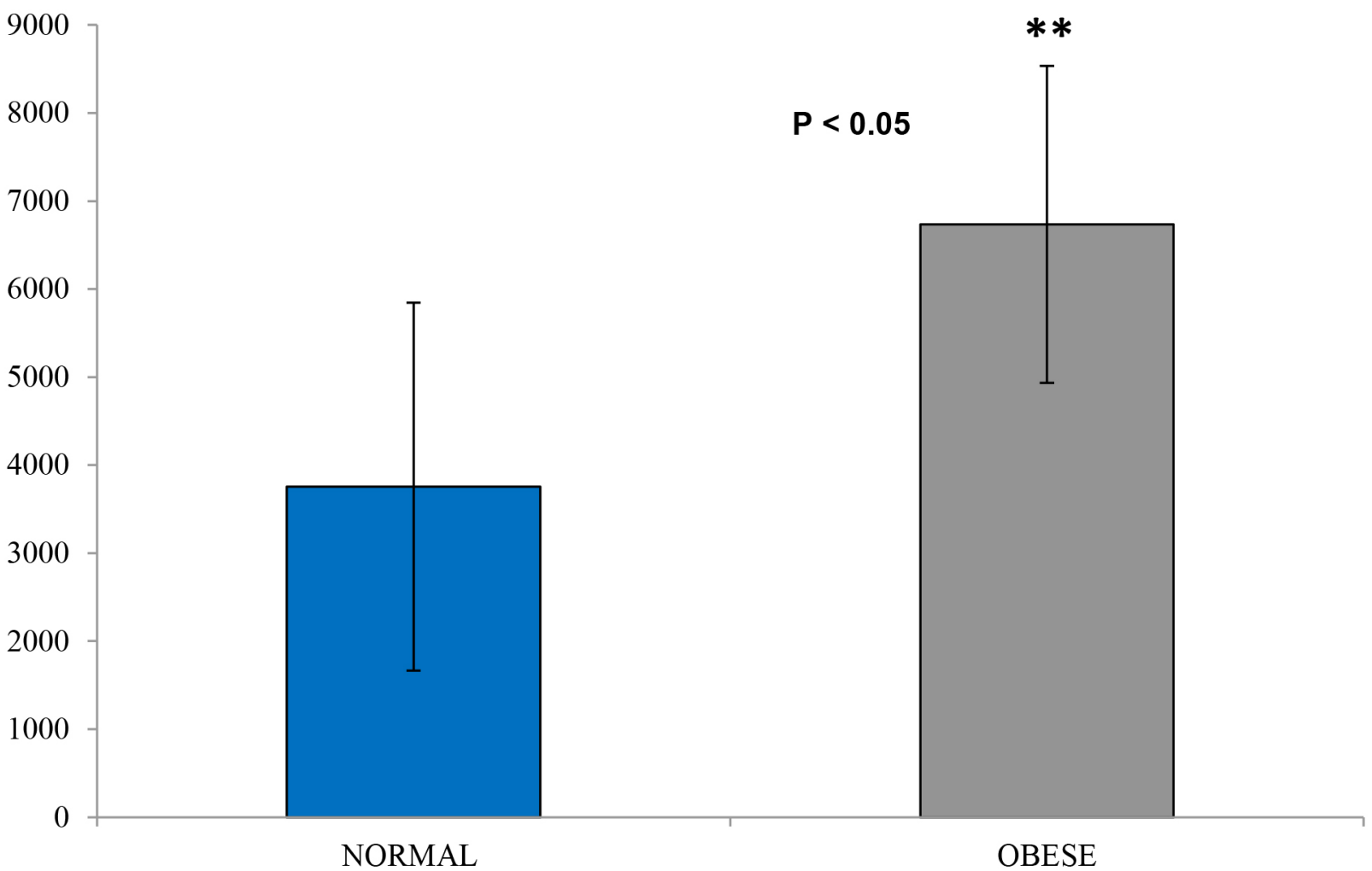

Figure 7. Insulin area under the curve 


\section{Discussion}

The subjects in this study were representative of a wide range of body weights characteristics of adult breeding females in our colony. Measurement of height and other body dimensions revealed that differences in weight were primarily attributed to body fat rather than to stature or lean body mass. Abdominal circumference and abdominal fat fold were the most highly correlated with total body fat. Furthermore, most monkeys had relatively normal fasting glucose and insulin concentrations. However, the glucose uptake among the obese group versus the lean group Rhesus monkeys was similar after the glucose challenge. The increase insulin concentrations observed in the obese group is also an indication of insulin resistance and a potential pre-diabetes state. The strong relationship between body fat and insulin levels has been previously reported for Rhesus monkeys and has been attributed to an effort to overcome peripheral resistance to insulin's action of lowering blood glucose levels [11]. The obese animals in this study exhibited hyperinsulinemia had normal fasting glucose levels and normal glucose tolerance. Similar findings were reported for a group of obese Rhesus males [7]. These studies showed that T2D in Rhesus monkeys is a progressive disorder with increased basal insulin secretion and impaired insulin response to glucose challenge as the earliest abnormalities. As Rhesus monkeys become diabetic, islet amyloid is abundant. Monkeys also exhibit age-related decreases in insulin sensitivity, insulin response to glucose, lean body mass, and energy expenditure, and obese animals' exhibit increased fasting triglycerides, increased fasting insulin, and impaired insulin responses to glucose. Obesity-related hyperinsulinemia and abnormal glucose tolerance have also been found in feral Rhesus monkeys.

The relevance of gender should also be considered as sex hormones such as estrogen and progesterone are reported to affect body weight and insulin resistance. Subsequently, both diabetes and cardiovascular disease will be influenced and monkeys with gestational diabetes have elevated glucose and insulin concentrations. The postmenopausal state is another factor associated with increased insulin resistance as well as an increase in body weight.

Both behavioural and physiologic factors are likely to contribute to the etiology of spontaneous obesity in captive macaques. As for humans, potential associations between various physiologic parameters and the social status of individual animals is an important consideration when assessing root causes of obesity in non-human primates. Cynomologus and other macaque species live in complex social hierarchies, in which there are dominant and subordinate animals. Social status influences access to food resources, which can affect body condition.

An additional consideration is the role of psychosocial stress in the etiology of diabetes. In humans, the current thinking is that much of the present-day T2D epidemic reflects insulin resistance secondary to visceral fat accumulation and a seemingly more direct relationship has been observed in animals. In non-human primates, the effects of stress on T2D are derived from animals living under relatively crowded social conditions. These individuals are less able to clear glucose challenge compared to their non-crowded counterparts. Furthermore, the experimental housing conditions can be manipulated to created interventions that disrupt species-typical lifestyle to effectively induce metabolic abnormalities [11, 12, and 14].

\section{Summary}

The obese Rhesus monkeys in our colony show evidence of insulin resistance and a potential pre-diabetes state. Our observations are similar to those previously reported for this species. Because of the similarities between adult-onset, obesity-related diabetes in Rhesus monkeys and humans, our specific long-term housing conditions will ensure the availability of suitable, aging monkeys to investigate the pathogenesis, risk factors, and some of the chronic diseases associated with T2D.

\section{Acknowledgement}

The authors are grateful to: The Delft animal technicians for their technical support and to Mrs. Chapman from Biomedical Research and Innovation Platform (BRIP) for the insulin analysis.

\section{Conflict of Interest Statement}

The authors declare that they have no conflict of interests. This work formed part of an in-house research project and was funded by the South African Medical Research Council.

\section{References}

1. Abdullah A, Peeters A, de Courten M, Stoelwinder J (2010) The magnitude of association between overweight and obesity and the risk of diabetes: a metaanalysis of prospective cohort studies. Diabetes Research \& Clinical Practice 89: 309-319.

2. Freemantle N, Holmes J, Hockey A, Kumar S (2008) How strong is the association between abdominal obesity and the incidence of type 2 diabetes? International Journal of Clinical Practice 62: 1391-1396 .

3. Després JP (2012) Body fat distribution and risk of cardiovascular disease: an update. Circulation 126: 1301-1313. [crossref]

4. Kahn SE, Hull RL, Utzschneider KM (2006) Mechanisms linking obesity to insulin resistance and type 2 diabetes. Nature 444: 840-846. [crossref]

5. Kaplan JR, Wagner JD (2006) Type 2 diabetes-an introduction to the development and use of animal models. ILAR J 47: 181-185. [crossref]

6. Wagner JD, Cann JA, Zhang L, Harwood HJ (2012) Jr. Diabetes and obesity research using nonhuman primates. Nonhuman primates in biomedical research Volume 2: Diseases. In: Abee CR, Mansfield K, Tardif SD, Morris T, editors. 2nd ed. Elsevier: Chapter 14: 699-732

7. Kemnitz JW, Francken GA (1986) Characteristics of spontaneous obesity in male rhesus monkeys. Physiol Behav 38: 477-483. [crossref]

8. Hansen BC, Bodkin NL (1986) Heterogeneity of insulin responses: Phases leading to type 2 (non-insulin-dependent) diabetes mellitus in the rhesus monkey. Diabetologia 29: 713-719.

9. Hansen BC (2001) Causes of obesity and consequences of obesity prevention in non-human primates and other animal models. In: International Textbook of Obesity. Edited Per Bjorntorp. John Wiley \& Son Ltd: Chapter 14: 182-201.

10. Cefalu WT (2006) Animal models of Type 2 Diabetes: Clinical presentation and pathophysiological relevance to the human condition. ILAR Journal 47: 186-197.

11. Wagner JE, Kavanagh K, Ward GM, Auerbach BJ, Harwood HJ Jr, et al. (2006) Old world nonhuman primate models of type 2 diabetes mellitus. ILAR J 47: 259-271. [crossref]

12. Harwood HJ Jr1, Listrani P, Wagner JD (2012) Nonhuman primates and other animal models in diabetes research. J Diabetes Sci Technol 6: 503-514. [crossref]

13. Clingerman KJ, Summers L (2005) Development of a body condition scoring system for nonhuman primates using Macaca mulatta as a model. Lab Animal 34: 31-36.

14. Bauer SA, Arndt TP, Leslie KE et al. (2011) Obesity in Rhesus and Cynomologus Macaques: A Comparative Review of the Condition and Its Implications for Research. Comparative Medicine 61: 514-526.

\section{Citation:}

Charon de Villiers, Joritha van Heerden1 and Johan Louw (2017) Insulin resistance in spontaneous obese Rhesus monkeys (Macaca mulatta) - A valuable animal model. Endocrinol Diabetes Metab J Volume 1(5): 1-6 